\title{
Recent developments in natural product-based drug discovery for tuberculosis
}

\author{
Maryline Dong ${ }^{1}$, Bernhard Pfeiffer ${ }^{1}$, and Karl-Heinz Altmann ${ }^{1}$ \\ ${ }^{1}$ ETH Zürich, Department of Chemistry and Applied Biosciences, Institute of Pharmaceutical Sciences, Vladimir-Prelog- \\ Weg 4, HCl H 405, CH-8093 Zürich, Switzerland \\ Corresponding author: Altmann, K-H. (karl-heinz.altmann@pharma.ethz.ch)
}

\begin{abstract}
Natural products (NPs) have been at the origin of several established drugs against tuberculosis (TB). Although the current clinical TB pipeline does not feature any candidates derived from new NP scaffolds, numerous novel NP or NP analogs have been discovered in the recent past with promising activity against Mycobacterium tuberculosis (Mtb) This includes newly discovered structures as well as known NP classes that had not been previously recognized to be active against Mtb. These compounds could help to replenish the dry clinical TB pipeline and, thus, contribute to improvements in the treatment of a devastating disease.
\end{abstract}

Keywords: drug discovery; natural products; tuberculosis.

Teaser: Natural products continue to serve as a prolific source of lead structures for drug discovery against TB.

\section{Introduction}

TB is a global threat to public health that is becoming ever more serious because of the widespread emergence of drug-resistant strains of the causative pathogen $M t b$ [1]. At the same time, TB-directed drug discovery has been subcritical for several decades. Although efforts towards the development of new TB drugs have re-intensified in the more recent past, resulting in the first approvals of two new TB drugs in more than 40 years (bedaquiline and delamanid) [2], there continues to be an urgent need for the discovery of novel, potent, and safe TB treatments.

NP have a rich history as lead compounds for drug discovery and development in different disease areas, but particularly so in the area of bacterial infections [3,4]. In fact, most clinically used antibiotics are NP or NP derivatives. Thus, it is no surprise that the first effective TB drug to be developed was the bacterial NP streptomycin, an aminoglycoside antibiotic isolated in 1944 from Streptomyces griseus by Waksman and Schatz (Table 1) [5]. Streptomycin and also other aminoglycosides to this day are important components of second-line drug combination regimens that are used in the treatment of multidrug-resistant (MDR)-TB [6]. Since the discovery of streptomycin, a multitude of NPs have been described in the literature to exhibit antimycobacterial activity. Most prominently, this includes rifamycin B, an ansamycin-type macrolactam that was first isolated from Streptomyces mediterranei in 1957; rifamycin B was the starting point for the development of the first-line TB drug rifampicin [5], which is a semisynthetic derivative of rifamycin B. In addition to rifampicin and aminoglycosides (streptomycin, amikacin, and kanamycin), further NP-based TB drugs are cycloserine and capreomycin, which are both part of second-line drug combinations [6].

Although the frequent occurrence of NP with the capacity to inhibit the growth of Mtb (at least in vitro) underscores the lead potential of NP for TB drug discovery, few NP/NP derivatives have been moved into drug development since the introduction of rifampicin in 1968. In fact, the current clinical development pipeline for TB includes only two NP derivatives (high-dose rifampicin and rifapentine-moxifloxacin) (www.newtbdrugs.org/pipeline/clinical), both of which are based on long known scaffolds [5]. In addition to the fact that drug development for TB has generally progressed at a slow pace, several specific issues contribute to the paucity of NP-based drug candidates in the clinical development pipeline for TB. These include: (i) the limited availability of material (because of low isolation yields or issues with source access); (ii) the lack of synthetic followup on promising leads, because of (perceived) synthetic difficulties; or (iii) the moderate activity of unmodified NP, whose minimum inhibitory concentration (MIC) are generally $<1 \mathrm{Mg} / \mathrm{ml}$. In addition, unmodified NP rarely have the pharmacokinetic properties needed for direct therapeutic use and, therefore, require chemical modification to make them suitable for clinical development. Only in vitro data are also available for most antimycobacterial NP and many of the corresponding literature reports do not include any selectivity and/or cytotoxicity data, which makes it impossible to judge the true lead potential of most NP with in vitro anti-Mtb activity. In fact, the toxicity risk associated with NP might be particularly pronounced, because many of them are produced by the source organisms to eliminate competing species or as a means of defense against predators.

In this review, we highlight some of the more recent discoveries in relation to antimycobacterial NP or NP derivatives. For the sake of brevity and conciseness, we focus on compounds with MICs $>3 \mathrm{Mg} / \mathrm{ml}$; although this particular potency cutoff is rather arbitrary, we firmly believe that NP with lower potency in vitro offer little promise as true leads for TB drug discovery (although, obviously, this cannot be excluded with certainty). However, even within this boundary, the list of compounds discussed here (Figure 1) is not exhaustive. The reader is referred to [7-9] for more inclusive reviews on antimycobacterial NP. 


\section{Peptides and depsipeptides}

\section{Griselimycins}

The cyclic peptide griselimycin (1) (Figure 1) was first reported in the literature in 1965 (as RP 11072) by Noufflard-Guy-Loe and Berteaux, who described its isolation from two species of Streptomyces, S. griseus and $S$. coelicus, and showed it to be active against mycobacteria [9]. The structural elucidation of 1 was then reported in a series of papers in 1969 [10] and 1971 [11]. After attempts to improve the unfavorable pharmacokinetic properties of the compound had failed at the time, interest in the compound ceased, especially after rifampicin became available for TB treatment in 1968 (according to [13]). Recently, however, the compound was revisited by Müller and coworkers [13] and they found that introducing alkyl substituents on the pyrrolidine ring of Pro ${ }^{8}$ led to a substantial increase in metabolic stability. In addition, these derivatives, such as cyclohexylgriselimycin (2) (Figure 1), showed enhanced antibiotic activity against $M t b$, with the MIC of 2 against $M t b$ H37Rv being 50-fold lower than for the parent NP $1(0.06 \mathrm{Mg} / \mathrm{ml}$ versus $1 \mathrm{Mg} / \mathrm{ml}$ for 1) [13]. MIC values for 2 against a series of different monoresistant $M t b$ strains were between 0.056 and $0.2 \mu \mathrm{g} / \mathrm{ml}$. Müller and coworkers were also able to elucidate the protein target of griselimycins as the DNA polymerase sliding clamp (DnaN) by means of resistant mutant sequencing. The genetic findings were further supported by subsequent surface plasmon resonance (SPR) measurements, which showed good selectivity of 2 for bacterial over human DnaN [13]. Ultimately, binding of griselimycin (1)/cyclohexylgriselimycin (2) to DnaN was firmly established by X-ray crystallography [13]. Cyclohexylgriselimycin (2) prevented bacterial growth and the formation of gross lung lesions in an acute mouse model of TB after oral administration. In a chronic model, the compound was shown to be equally effective as rifampicin [13].

\section{Cyclomarins}

In 1999, the groups of Clardy and Fenical reported the isolation of a small family of cycloheptapeptides from the marine streptomycete CNB-982, termed 'cyclomarins A-C' (Figure 1) [14]. Although these compounds were noteworthy at the time because of their anti-inflammatory activity, the major metabolite cyclomarin A (3) was shown by a group at Novartis in 2011 to be active against $M t b$, with cidal concentrations of $0.3 \mu \mathrm{M}$ and $2.5 \mu \mathrm{M}$ against bacteria in culture broth medium and in human-derived macrophages, respectively [15]. The compound also inhibited the growth of nonreplicating, intracellular bacteria in macrophages (90\% kill after exposure to 2.5 $\mathrm{mM}$ of 3 for 5 days) and was found to retain activity against different MDR strains of $M t b$, although no MICs were reported for this activity [15]. No spontaneous resistant $M t b$ mutants could be raised upon exposure of $M t b$ to cyclomarin A (3), suggesting a mutation frequency of $>10^{-9}$. Using a proteomic approach with Mycobacterium bovis BCG as a model organism, the protein target of cyclomarin A (3) was identified to be the ATPase ClpC1 (Rv3596c), the regulatory subunit of the caseinolytic Clp protease. In agreement with these findings, cyclomarin A (3) was found to specifically increase proteolysis in Mycobacterium smegmatis, as demonstrated by the significantly increased degradation rate of GFP tagged with a Clp recognition sequence, compared with the unmodified protein (40\% versus $10 \%$ degradation, respectively, after treatment with $0.3 \mu \mathrm{M} 3$ for $4 \mathrm{~h}$ ) [15]. The equilibrium dissociation constant for the complex between cyclomarin (3) and ClpC1 was determined as $16 \mathrm{nM}$ for a 1:1 stoichiometry; the binding of 3 to isolated ClpC1 was not competitive with ATP binding. Of three cyclomarin-type structures studied in [15] [including cyclomarin A (3)], only those two that showed activity against $M t b$ were able to prevent binding of 3 to $\mathrm{ClpC} 1$ in an $M t b$ cell lysate. The Novartis group subsequently also reported significant antimalarial activity of 3 , because of selective, nanomolar inhibition of the diadenosine triphosphate hydrolase (PfAp 3 Ase) of Plasmodium falciparum $\left(\mathrm{IC}_{50}=4 \mathrm{nM}\right)$, whereas the human homolog of PfAp 3 Ase (hFHIT; human fragile histidine triad) was not affected [16]. In 2016, Barbie and Kazmaier reported the synthesis of desoxycyclomarin C (6), which has the $\beta^{-}$ hydroxy-L-Trp in cyclomarin C (5) replaced with L-Trp. Interestingly, this compound retained antimycobacterial activity, with an MIC of $2 \mathrm{Mg} / \mathrm{ml}$ versus $0.5 \mathrm{Mg} / \mathrm{ml}$ for cyclomarin $\mathrm{C}$ (5) (the tested strain is not indicated in the paper) [17]. This latter study was performed in the context of a more comprehensive program by the Kazmaier group on the synthesis of cyclomarins and cyclomarin analogs. This work could open a path to synthetic cyclomarin analogs with improved pharmacokinetic properties over the NPs, which are cited by Kazmeier et al. as being extremely susceptible to metabolic degradation and, therefore, cannot serve as drug candidates. (No published original data on the PK properties of cyclomarins are available, however.) As demonstrated by the work on griselimycins, such improvements could be feasible [13].

\section{Ecumicin}

Gao et al. recently reported the isolation of ecumicin (7) (Figure 1) [18], a head-to-side-chain cyclic tridecapeptide comprising mainly $N$-methylated amino acids, from Nonomuraea sp. MJM5123. Given its particular structural features (large fraction of $N$-methyl/D-configured amino acids), ecumicin (7) is highly resistant to enzymatic degradation [19]. Similar to cyclomarin A (3), ecumicin (7) specifically binds to ClpC1 of Mtb [19,20] and its in vitro anti-Mtb activity profile is comparable with that of current first-line TB drugs. Thus, the compound exhibits MIC values against sensitive $M t b$ strains of $0.16-0.58 \mathrm{Mg} / \mathrm{ml}$ and it retains activity against monodrug-resistant (MICs $<0.12-0.31 \mathrm{Mg} / \mathrm{ml}$ ) as well as MDR and extensively drug-resistant (XDR) strains of $M t b(\mathrm{MICs}<0.12-0.31 \mathrm{Mg} / \mathrm{ml}$ and $0.31-0.62 \mathrm{Mg} / \mathrm{ml}$, respectively). Ecumicin (7) is essentially nontoxic to mammalian cells (IC50 values of $<63 \mu \mathrm{M}$ and $<32 \mu \mathrm{M}$ against Vero and J774 cells, respectively) [19] and it has also been shown to be active in a mouse model of TB after subcutaneous administration [19]. 


\section{Lassomycin}

The 'lasso' hexadecapeptide lassomycin (8) was recently isolated from the Actinomycete Lentzea kentuckyensis spp. IO0009804 by Garvish et al. (Figure 1) [21]. Similar to ecumicin (7) and cyclomarin A (3), 8 has been reported to target and bind to $\mathrm{ClpC} 1$, which appears to be emerging as a (clinically) unexplored, high-potential target for new TB drug discovery [19]. Lassomycin (8), similar to ecumicin (7), leads to a several-fold increase in the ATPase activity $\mathrm{ClpC} 1$, but in contrast to the latter, which causes excessive protein degradation, 8 inhibits ClpP1P2dependent proteolysis [21]. As indicated above, excessive protein degradation is also triggered by cyclomarin A (3); no data have been reported for the effect of 3 on the ATPase activity of ClpC1. Lassomycin (8) inhibits $M t b$ growth in vitro with MICs in the range of $0.8-3 \mathrm{Mg} / \mathrm{ml}$ and its activity extends across a variety of $M t b$ strains, including MDR and XDR isolates [21]. However, more recently, the biological evaluation of synthetic lassomycin (8) (based on the structure reported in [21]) and its C-terminal amide failed to reveal any activity against $M t b$ for either compound [22]. Although the reasons for this discrepancy have not yet been fully established, temperaturedependent NMR measurements on synthetic lassomycin (8) suggest that natural lassomycin (8) exists in a threaded conformation that does not unfold upon heating to $40^{\circ} \mathrm{C}$, contrary to the original proposal that the peptide would exist in an unthreaded state [21].

\section{Teixobactin}

In 2015, Ling et al. reported the discovery of the head-to-side chain cyclic depsipeptide teixobactin (9) (Figure 1) [23], based on a screen for antimicrobial activity against Staphylococcus, aureus of extracts obtained from uncultured soil bacteria [24]; the species producing teixobactin (9) was provisionally named Eleftheria terrae. The compound was subsequently found to exhibit excellent activity against Gram-positive pathogens, including drugresistant strains [23] and also to inhibit $M t b \mathrm{H} 37 \mathrm{Rv}$ with an MIC of $0.125 \mathrm{Mg} / \mathrm{ml}$. Teixobactin (9) blocks cell wall biosynthesis in vitro by binding to a highly conserved motif on the peptidoglycan precursor lipid II and to cell wall teichoic acid precursor lipid III. Given its lack of cytotoxicity against NIH/3T3 and HepG2 cells even at $100 \mathrm{Mg} / \mathrm{ml}$ (the highest concentration tested), teixobactin (9) is a promising lead for the development of new antibiotics. In fact, the compound has shown in vivo activity against $S$. aureus and Streptococcus pneumoniae [23]. However, it is unlikely to be orally bioavailable, which clearly limits its potential as a TB drug candidate per se.

Initial structure-activity relation (SAR) studies with synthetic teixobactin analogs $[25,26]$ have shown that the $L$-allo-enduracididine residue can be replaced by either Arg or, preferably, Lys. By contrast, modifications of the Nterminal tail lead to a loss of activity.

\section{Pyridomycin}

Pyridomycin (10) (Figure 1) is a bacterial NP that was first isolated in 1953 by Maeda and coworkers from Streptomyces albidofuscus (S. pyridomyceticus) [27]. Although the compound was shown to exhibit significant in vitro antimycobacterial activity (MIC $50.3 \mathrm{Mg} / \mathrm{ml}, \mathrm{H} 37 \mathrm{Rv}$ ) and to display low cytotoxicity [28], no attempts at the further profiling of pyridomycin (10) or the elucidation of its SAR were reported in the literature until recently. The in vitro antimycobacterial activity of pyridomycin (10) against actively growing bacteria was reconfirmed in 2012 by Hartkoorn et al. [29], although the compound was not active against nonreplicating $M$. tuberculosis in the streptomycin-starved $18 \mathrm{~b}$ (ss18b) model. In a subsequent step, pyridomycin-resistant mutants of Mtb were selected. Whole-genome sequencing and genetic validation then revealed that pyridomycin (10) was targeting the NADHdependent enoyl-[acyl-carrier-protein] reductase InhA, which is an essential enzyme for cell wall biosynthesis in $M t b$ and is the target of the clinical TB drugs isoniazid and ethionamide [30,31]. As shown by X-ray crystallography, pyridomycin binds to InhA in a unique configuration, because the compound spans both the NADH as well as the substrate-binding sites of the enzyme. This binding mode is distinct from all other known direct InhA inhibitors, which bind to the substrate-binding site of the protein [32,33]. As a consequence of its direct inhibition of InhA, pyridomycin (10) retains activity against InhA-resistant strains of $M t b$, which are mostly characterized by mutations in the isoniazid-activating enzyme KatG rather than in InhA itself.

So far, only one total synthesis of pyridomycin has been reported in the literature, by Kinoshita et al. in 1989 [34]. One of the major difficulties in this synthesis was the establishment of the structurally unique enol-ester moiety that is part of the pyridomycin macrocycle. To bypass these difficulties, dihydropyridomycins 11 and 12 have been studied; these have an iso-propyl substituent attached to C2 by a single, rather than a double bond [35]. Intriguingly, the $2 R$ analog 11 retained most of the activity of natural pyridomycin (a fourfold increase in MIC against $\mathrm{H} 37 \mathrm{Rv}$ ), thus demonstrating that the enol-ester double bond is not a prerequisite for the anti-Mtb activity of 10 . The $2 S$ isomer 12 was 32 -fold less potent than the NP. Other pyridomycin analogs that are based on the dihydropyridomycin core structure are currently being investigated.

\section{Nonpeptidic structures}

\section{Tryptanthrins}

Tryptanthrin (13) (Figure 1) is a natural indolo[2,1-b]quinazoline-6,12-dione that was first isolated in 1979 by Honda and coworkers [36] from the indigo plant Strobilanthes cusia O. Kuntze (Acanthaceae). In 1998, Mitscher and Baker [37] described the antiTB activity of tryptanthrin and several analogs, with PA-505 (14) and PA-510 (15) as the most promising compounds at the time, but little efficacy was observed in a mouse model of acute TB (without this being commented on). 
Tryptanthrin (13) is poorly water soluble (5.4 MM) and, because of its planar structure, it might also act as a DNA intercalator, which would lead to cytotoxicity. In light of these shortcomings, Hwang et al. [38] recently embarked on the design and synthesis of a series of new tryptanthrin analogs with less planar structures and incorporating solubilizing groups. The most promising of these compounds, 16 (Figure 1), exhibited MICs of 0.5 $\mathrm{mg} / \mathrm{ml}$, in the microplate Alamar Blue assay (MABA) against $\mathrm{H} 37 \mathrm{Rv}$, and $11.5 \mathrm{mg} / \mathrm{ml}$, in a low-oxygen recovery assay (LORA); the oral bioavailability of 16 in rats was $30 \%$. However, the compound failed to show any efficacy in vivo in a murine model of acute TB. The discrepancy between the in vitro and in vivo data could not be explained, but could result from insufficient plasma and/or target tissue concentrations. We have included this example, because the study highlights the fact that potent in vitro anti-Mtb activity is an insufficient measure for the true lead potential of a compound for drug discovery. Although this is in fact a trivial conclusion, there is a vast amount of literature on compounds (including some of those discussed in here), which have been claimed to be promising leads for TB-directed drug discovery solely based on MIC values. The results of Hwang et al. on their tryptanthrin analogs put these claims in perspective.

\section{Tiacumicin B}

The antimicrobial glycosylated macrolide tiacumicin B (17) (Figure 1) was isolated in 1987 from the soil bacterium Dactylosporangium aurantiacum subsp. hamdenensis subsp. nov. AB718C-41 [39] and was later shown [40] to be identical with the previously reported lipiarmycin A3 [41]. Tiacumicin B (17) is a bacterial RNA polymerase inhibitor [42] and has been approved (as fidaxomicin; Dificlir ${ }^{\circledR}$, Dificid $\AA$ ) for the treatment of infections with Clostridium difficile [43]. The compound exhibits activity against a broad range of Gram-positive organisms [44]; more importantly, in the context of this review, it was also found in 2008 to be a potent inhibitor of $M t b$, including several MDR-Mtb strains (MICs of 0.01-1 Mg/ml) [45]. The major obstacle for the development of tiacumicin B (17) for TB therapy is its low bioavailability and acid sensitivity [46]. Recently, two independent syntheses of the tiacumicin B aglycone [47,48] and one total synthesis of the complete NP [49] were described, which provide a basis for SAR studies on synthetic tiacumicin analogs that might eventually lead to compounds with a property profile suitable for oral therapy. As illustrated by the recent advances on the pharmacokinetic profile of griselimycins, such improvements are not impossible to achieve.

\section{Caprazamycins}

Caprazamycins (CPZs) (Figure 1) are liponucleoside antibiotics that were first isolated in 2003 by Igarashi et al. from Streptomyces sp. MK730-62F2 [50]. They are generally active against acid-fast bacteria, including $M t b$, with CPZ B (18) being the most potent antimycobacterial agent ( $\mathrm{MIC}$ against $M t b \mathrm{H} 37 \mathrm{Rv}=3.13 \mathrm{Mg} / \mathrm{ml}$ ) [51]. The antibiotic activity of CPZs results from inhibition of the biosynthetic enzyme MraY (translocase I), which is essential for bacterial cell growth. Although CPZ B (18) showed no significant toxicity in mice, its difficult isolation process (the compound has to be separated from a complex mixture by HPLC) and its poor water solubility made it unsuitable for drug development.

Treatment of the mixture of CPZs A-G with aqueous acid leads to cleavage of the ester-linked side chain and the release of the (inactive) common core structure, which is referred to as 'caprazene' (19) (CPZEN) [52]. The investigation of various non-natural ester derivatives of CPZEN led to the discovery of CPZEN-45 (20) as the most active CPZEN analog; the compound exhibits an MIC against $M t b \mathrm{H} 37 \mathrm{Rv}$ of $1.56 \mathrm{Mg} / \mathrm{ml}$, is active against MDR and XDR $M t b$ strains, and shows excellent water solubility (>200 mg/ml). [MIC values against monodrug-resistant $M t b$ strains have only been published in various patent applications (e.g., [53]) and are reported to be around $6 \mu \mathrm{g} / \mathrm{ml}$. Intriguingly, CPZEN-45 (20) was also active against CPZ-resistant Bacillus subtilis strains, but was not effective against a strain overexpressing undecaprenyl-phosphate-GlcNAc-1-phosphate transferase (TagO), an enzyme involved in the synthesis of teichoic acid. At the same time, a mutation was found in the tagO gene of a spontaneous CPZEN-45-resistant strain, suggesting that the mechanism underlying the antibiotic activity of CPZEN-45 (20) in Bacillus subtilis was the inhibition of TagO. This was subsequently confirmed by Ishizaki et al., who showed that CPZEN-45 inhibited glycerol incorporation in B. subtilis using radiolabeled precursors and evaluated its activity using the sonicated cell lysate of B. subtilis strains overexpressing MraY or TagO [54]. Furthermore, and more importantly in the context of this review, more recent work reported by Ishizaki et al. also suggests that CPZEN-45 (20) inhibits WecA, which is the ortholog of TagO in Mtb, with an $\mathrm{IC}_{50} \mathrm{of} 4.4 \mathrm{ng} / \mathrm{ml}$ (in membrane fractions of $\left.M t b \mathrm{mc}^{2} 6230\right)$.

Neither MraY nor WecA are targeted by current TB drugs and, thus, the successful clinical development of inhibitors of these enzymes would lead to TB drugs with an entirely new mode of action. Such compounds would be devoid of any (mechanism-related) cross-resistance with existing drugs. CPZEN-45 (20) is currently in preclinical development for TB.

\section{Diazaquinomycins}

Diazaquinomycins (DAQs) are biologically active secondary metabolites with a diaza-anthracene structure (Figure 1). Several representatives of this class have been investigated as antibiotics and also as anticancer agents $[55,56]$. However, it was only recently that Mullowney et al. discovered the potent antimycobacterial activity of some DAQs, in the context of the isolation of the new DAQs E-G from Streptomyces sp. [57] and H and J from Micromonospora sp. [58]. DAQH (21) and DAQJ (22) exhibited MICs of 0.04 and $0.07 \mathrm{Mg} / \mathrm{ml}$, respectively, against Mtb H37Rv; potent anti-Mtb activity was also found for DAQA (23) and DAQE (24) (MICs of 0.1 and $0.04 \mathrm{Mg} / \mathrm{ml}$, respectively). 
DAQA (23) was also found to be active against nonreplicating bacteria (MIC of $0.72 \mu \mathrm{g} / \mathrm{ml}$ in the LORA assay) and it retained high potency against a panel of various monodrug-resistant strains of $M t b(\mathrm{MIC}$ values $<1 \mu \mathrm{g} / \mathrm{ml})$, suggesting absence of cross-resistance with current TB agents [58]. Mullowney et al. [58] also re-evaluated the cytotoxicity of DAQA (23) and found it to be well tolerated compared with earlier studies [56]. DAQs have been reported to inhibit thymidylate synthase from Ehrlich ascites and it has been implied that this enzyme is the target of DAQs in both cancer cells as well as Gram-positive bacteria [56]. However, more recent studies have demonstrated that thymidylate synthase is not the target of DAQs in Mtb [58].

\section{Spectinamides}

Instead of screening for agents that inhibit $M t b$ and trying to optimize the hits produced, an alternative strategy to develop new TB drugs is the repurposing of existing antibacterials that are known to be safe and effective against other infections, but that have not been used extensively (or not at all) to treat TB. A prime example for how such a strategy can lead to highly interesting, new anti-Mtb agents is the work recently reported by Lee et al. [59] on new spectinomycin derivatives.

Spectinomycin (25) (Figure 1) itself is a bacterial NP that has a long history of safe use for the treatment of gonorrhea in patients who are allergic (or resistant) to penicillins. Based on the careful literature analysis of available SAR data and the construction of a homology model of the purported spectinomycin binding site in the Mtb 16S ribosomal subunit (derived from the structure of the Escherichia coli 30S ribosomal subunit/spectinomycin complex), Lee et al. concluded that the replacement of the $3^{\prime}$-keto group in spectinomycin by an acylated amino group should be well tolerated for an $R$ configuration of the newly created stereocenter at C3. Using spectinomycin as the starting material, a series of such analogs, termed 'spectinamides' (26) (Figure 1) was produced in a convergent four-step (semi)synthesis. The optimization process, which heavily relied on the use of the above homology model for the selection of improved acyl residues, finally led to three candidate molecules with MIC values against $M t b \mathrm{H} 37 \mathrm{Rv}$ of $0.8-1.6 \mathrm{Mg} / \mathrm{ml}$; these compounds were also active against MDR and XDR clinical isolates (with MICs against different monodrug-resistant $M t b$ strains between 0.8 and $6.3 \mu \mathrm{g} / \mathrm{mL}$ ) [59]. For one variant of 26 [R 5 2-(5-chloropyridin-2-yl)acetyl; Figure 1], activity was also assessed against hypoxic bacilli and the compound was determined to be 'highly active' $(<2 \%$ viable bacteria at $10 \mu \mathrm{g} / \mathrm{mL}$ of 26$)$. The greatly improved anti$M t b$ activity of these compounds over the parent NP was found to result from the reduced susceptibility of the former to drug efflux. The importance of drug efflux for the activity of spectinamides (26) was initially suggested by the lack of a correlation between their antiTB activity and the inhibition of mycobacterial ribosomal translation, and was subsequently confirmed by experiments with knockout mutants for the multidrug transporter Rv1258c, which is known to induce intrinsic spectinomycin resistance [60]. Given their different ribosomal binding sites, spectinamides 26 lack cross-resistance with other protein synthesis inhibitors, such as streptomycin, amikacin, kanamycin, capreomycin, or linezolid. The optimized spectinamides 26 exhibit favorable pharmacokinetic profiles and they are not cytotoxic. They have also shown excellent in vivo activity in both acute and chronic TB models in mice.

\section{Concluding remarks}

NPs have contributed in a major way to the currently available portfolio of drugs for the treatment of TB, with one first-line drug and several second-line agents being either NP themselves or being derived from a NP lead. At the same time, no drug derived from a new NP scaffold has been approved for clinical use since the first introduction of rifamycin-based drugs during the 1960s. However, this situation is nothing more than a reflection of several decades of subcritical efforts on TB drug development in general, which has led to a serious shortage of new drugs and drug candidates in clinical development against this devastating disease. In fact, several NPs have been found over the past few years to exhibit promising activity against the causative pathogen $M t b$. In light of continuous advances in NP research at various levels (isolation techniques, methods for structural characterization, combinatorial biosynthesis, mutasynthesis, activation of silent gene clusters, synthetic methodology, etc.), we will likely continue to see the emergence of new structures with interesting anti-Mtb activity and acting through new modes of action. Obviously, there are many challenges that need to be overcome on the way from a bioactive NP to a viable therapeutic; one of the most critical issues often being the achievement of sufficient metabolic stability and oral bioavailability. However, as illustrated by the example of cyclohexylgriselimycin, improvements in these parameters is possible, even by limited structural modification of the original NP lead. Also, the poor economic status of those countries and regions that are most affected by the disease imposes severe restrictions on the affordable cost of goods for drugs against TB. Despite these challenges, we believe that NP can continue to serve as source of new and innovative drugs against TB, if sufficient funding is made available for research and development.

Acknowledgment

M.D. is supported by the Swiss National Science Foundation (SNF), grant number 200021_149253.

References

1 WHO (2015) Global Tuberculosis Report (20th edn), WHO

2 Zumla, A.I. et al. (2014) New antituberculosis drugs, regimens, and adjunct therapies: needs, advances, and future prospects. Lancet Infect. Dis. 14, 327-340

3 Newman, D.J. and Cragg, G.M. (2016) Natural products as sources of new drugs from 1981 to 2014. J. Nat. Prod. 79, 629-661

4 Patridge, E. et al. (2016) An analysis of FDA-approved drugs: natural products and their derivatives. Drug Discov. Today 21, 204-207 
5 Ahmad, Z. et al. (2011) History of drug discovery: early evaluation studies and lessons learnt from them. Antituberculosis Chemother. Prog. Respir. Res. 40, 2-9

6 WHO (2010) Guidelines for Treatment of Tuberculosis (4th edn), WHO

7 Copp, B.R. and Pearce, A.N. (2007) Natural product growth inhibitors of Mycobacterium tuberculosis. Nat. Prod. Rep. 24, 278-297

8 García, A. et al. (2012) Recent advances in antitubercular natural products. Eur. J. Med. Chem. 49, 1-23

9 Sieniawska, E. (2015) Targeting mycobacterial enzymes with natural products. Chem. Biol. 22, 1288-1300

10 Noufflard-Guy-Loe, H. and Berteaux, S. (1965) Experimental antituberculous action of a new antibiotic. Rev. Tuberc. Pneumol. 29, 301-326

11 Terlain, B. and Thomas J.P. (1969) Structure of griselimycin, a polypeptide antibiotic extracted from streptomyces cultures. $C R$ Acad. Sci $C$ Chim. 269, 1546-1549

12 Terlain, B. and Thomas J.P. (1971) Constitution of griselimycin, a polypeptide antibiotic extracted from Streptomyces cultures. II. Structure of griselimycin. Bull. Soc. Chim. Fr. 6, 2357-2362

13 Kling, A. et al. (2015) Targeting DnaN for tuberculosis therapy using novel griselimycins. Science 348, 1106-1112

14 Renner, M.K. et al. (1999) Cyclomarins A-C, new antiinflammatory cyclic peptides produced by a marine bacterium (Streptomyces sp.). J. Am. Chem. Soc. 121, 11273-11276

15 Schmitt, E.K. et al. (2011) The natural product cyclomarin kills Mycobacterium Tuberculosis by targeting the ClpC1 subunit of the caseinolytic protease. Angew. Chem. Int. Ed. 50, 5889-5891

16 Bürstner, N. et al. (2015) Gift from nature: cyclomarin A kills mycobacteria and malaria parasites by distinct modes of action. ChemBioChem 16, 2433-2436

17 Barbie, P. and Kazmaier, U. (2016) Total synthesis of desoxycyclomarin C and the cyclomarazines A and B. Org. Biomol. Chem. 14, 60556064

18 Gao, W. et al. (2014) Discovery and characterization of the tuberculosis drug lead ecumicin. Org. Lett. 16, 6044-6047

19 Gao, W. et al. (2015) The cyclic peptide ecumicin targeting ClpC1 is active against Mycobacterium tuberculosis in vivo. Antimicrob. Agents Ch. $59,880-889$

20 Lee, H. et al. (2016) Anti-tuberculosis lead molecules from natural products targeting Mycobacterium tuberculosis ClpC1. J. Ind. Microbiol. Biotechnol. 43, 205-212

21 Garvish, E. et al. (2014) Lassomycin, a ribosomally synthesized cyclic peptide, kills Mycobacterium tuberculosis by targeting the ATPdependent protease ClpC1P1P2. Chem. Biol. 21, 509-518

22 Lear, S. et al. (2016) Total chemical synthesis of lassomycin and lassomycin-amide. Org. Biomol. Chem. 14, 4534-4541

23 Ling, L.L. et al. (2015) A new antibiotic kills pathogens without detectable resistance. Nature 517, 455-459; Erratum: Nature 520, 388

24 Nichols, D. et al. (2010) Use of ichip for high-throughput in situ cultivation of 'uncultivable' microbial species. Appl. Environ. Microbiol. 76, 2445-2450

25 Yang, H. et al. (2016) Elucidation of the teixobactin pharmacophore. ACS Chem. Biol. 11, 1823-1826

26 Abdel, M. et al. (2016) Re-evaluation of the N-terminal substitution and the D-residues of teixobactin. RSC Adv. 6, 73827-73829

27 Maeda, K. et al. (1953) A new antibiotic, pyridomycin. J. Antibiot. (Tokyo) 6, 140

28 Hartkoorn, R.C. et al. (2012) Towards a new tuberculosis drug: pyridomycin - nature's isoniazid. EMBO Mol. Med. 4, 1032-1042

29 Hartkoorn, R.C. et al. (2014) Pyridomycin bridges the NADH- and substrate-binding pockets of the enoyl reductase InhA. Nat. Chem. Biol. $10,96-98$

30 Banerjee, A. et al. (1994) InhA, a gene encoding a target for isoniazid and ethionamide in Mycobacterium tuberculosis. Science 263, 227230

31 Vilchèze, C. et al. (2006) Transfer of a point mutation in Mycobacterium tuberculosis InhA resolves the target of isoniazid. Nat. Med. 12, 1027-1029

32 Pan, P. and Tonge, P.J. (2012) Targeting InhA, the FasII Enoyl-ACP reductase: SAR studies on novel inhibitors scaffolds. Curr. Top. Med. Chem. 12, 672-693

33 Martínez-Hoyos, M. et al. (2016) Antitubercular drugs on old target: GSK693 as a promising InhA direct inhibitor. EBioMedicine 8, 291301

34 Kinoshita, M. et al. (1989) Total synthesis of pyridomycin. Tetrahedron Lett. 30, 7419-7422

35 Horlacher, O. et al. (2013) Synthesis and antimycobacterial activity of 2,1-dihydropyridomycins. ACS Med. Chem. Lett. 4, 264-268

36 Honda, G. and Tabata, M. (1979) Isolation of antifungal principle tryptanthrin, from Strobilanthes cusia O. Kuntze. Planta. Med. 36, 85-86

37 Mitscher, L.A. and Baker, W. (1998) Tuberculosis: a search for novel therapy starting with natural products. Med. Res. Rev. 18, 363-374

38 Hwang, J.M. et al. (2013) Design, synthesis, and structure-activity relationship studies of tryptanthrins as antitubercular agents. J. Nat. Prod. 76, 354-367

39 Hochlowski, J.E. et al. (1987) Tiacumicins, a novel complex of 18-membered macrolides II. Isolation and structure determination. $J$. Antibiot. 40, 575-588

40 Bedeschi, A. et al. (2014) The co-identity of lipiarmycin A3 and tiacumicin B. Nat. Prod. Commun. 9, 237-240

41 Coronelli, C. et al. (1975) Lipiarmycin, a new antibiotic from Actinoplanes. II. Isolation, chemical, biological and biochemical characterization. J. Antibiot. 28, 253-259

42 Artsimovitch, I. et al. (2012) Fidaxomicin is an inhibitor of the initiation of bacterial RNA synthesis. Clin. Infect. Dis. 55, 127-131

43 Lucado, J. et al. (2012) Clostridium difficile Infections (CDI) in Hospital Stays, 2009. HCUP Statistical Brief \#124

44 Goldstein, E.J.C. et al. (2012) Antimicrobial activities of fidaxomicin. Clin. Infect. Dis. 55, 143-148

45 Kurabachew, M. et al. (2008) Lipiarmycin targets RNA polymerase and has good activity against multidrug-resistant strains of Mycobacterium tuberculosis. J. Antimicrob. Chemother. 62, 713-719

46 Sears, D. et al. (2012) Fidaxomicin attains high fecal concentrations with minimal plasma concentrations following oral administration in patients with Clostridium difficile infection. Clin. Infect. Dis. 55, S116-S120

47 Miyatake-Ondozabal, H. et al. (2015) Total synthesis of the protected aglycon of fidaxomicin (Tiacumicin B, Lipiarmycin A3). Angew. Chem. Int. Ed. 54, 1933-1936

48 Glaus, F. and Altmann, K-H. (2015) Total synthesis of the tiacumicin B (lipiarmycin A3/fidaxomicin) aglycone. Angew. Chem. Int. Ed. 54, $1937-1940$

49 Kaufmann, E. et al. (2015) Total synthesis of the glycosylated macrolide antibiotic fidaxomicin. Org. Lett. 17, 3514-3517

50 Igarashi, M. et al. (2005) Caprazamycins, novel lipo-nucleoside antibiotics, from Streptomyces sp. II. Structure elucidation of caprazamycins. J. Antibiot. 58, 327-337

51 Igarashi, M. et al. (2003) Caprazamycin B, a novel anti-tuberculosis antibiotic, from Streptomyces sp. J. Antibiot. 56, 580-583

52 Takahashi, Y. et al. (2013) Novel semisynthetic antibiotics from caprazamycins A-G: caprazene derivatives and their antibacterial activity. J. Antibiot. 66, 171-178

53 Takahashi, Y. et al. Microbial Chemistry Research Foundation (JP)/Infectious Disease Research Institute (US). Anti-XDR-TB drug, antiMDR-TB drug, and combination anti-tuberculosis drug. US9040502 B2 
54 Ishizaki, Y. et al. (2013) Inhibition of the first step in synthesis of the mycobacterial cell wall core, catalysed by the GlcNAc-1-phosphate transferase WecA, by the novel caprazamycin derivative CPZEN-45. J. Biol. Chem. 288, 30309-30319

55 Omura, S. et al. (1983) The structures of diazaquinomycins A and B, new antibiotic metabolites. Tetrahedron Lett. 24, 3643-3646

56 Tsuzuki, K. et al. (1989) Synthesis and biological activity of analogs of diazaquinomycin A, a new thymidylate synthase inhibitor. $J$. Antibiot. 42, 727-737

57 Mullowney, M.W. et al. (2014) Diazaquinomycins E-G, novel diaza-anthracene analogs from a marine-derived Streptomyces sp. Mar. Drugs $12,3574-3586$

58 Mullowney, M.W. et al. (2015) Diaza-anthracene antibiotics from freshwater-derived Actinomycete with selective antibacterial activity towards Mycobacterium tuberculosis. ACS Infect. Dis. 1, 168-174

59 Lee, R.E. et al. (2014) Spectinamides: a new class of semisynthetic antituberculosis agents that overcome native drug efflux. Nat. Med. 20, $152-158$

60 Ramon-Garcia, S. et al. (2012) Functional and genetic characterization of the Tap efflux pump in Mycobacterium bovis BCG. Antimicrob. Agents Chemother. 56, 2074-2083

Figure 1. Molecular structures of natural products or natural product derivatives that have recently been shown to exhibit anti-Mycobacterium tuberculosis (Mtb) activity. 
Figure 1

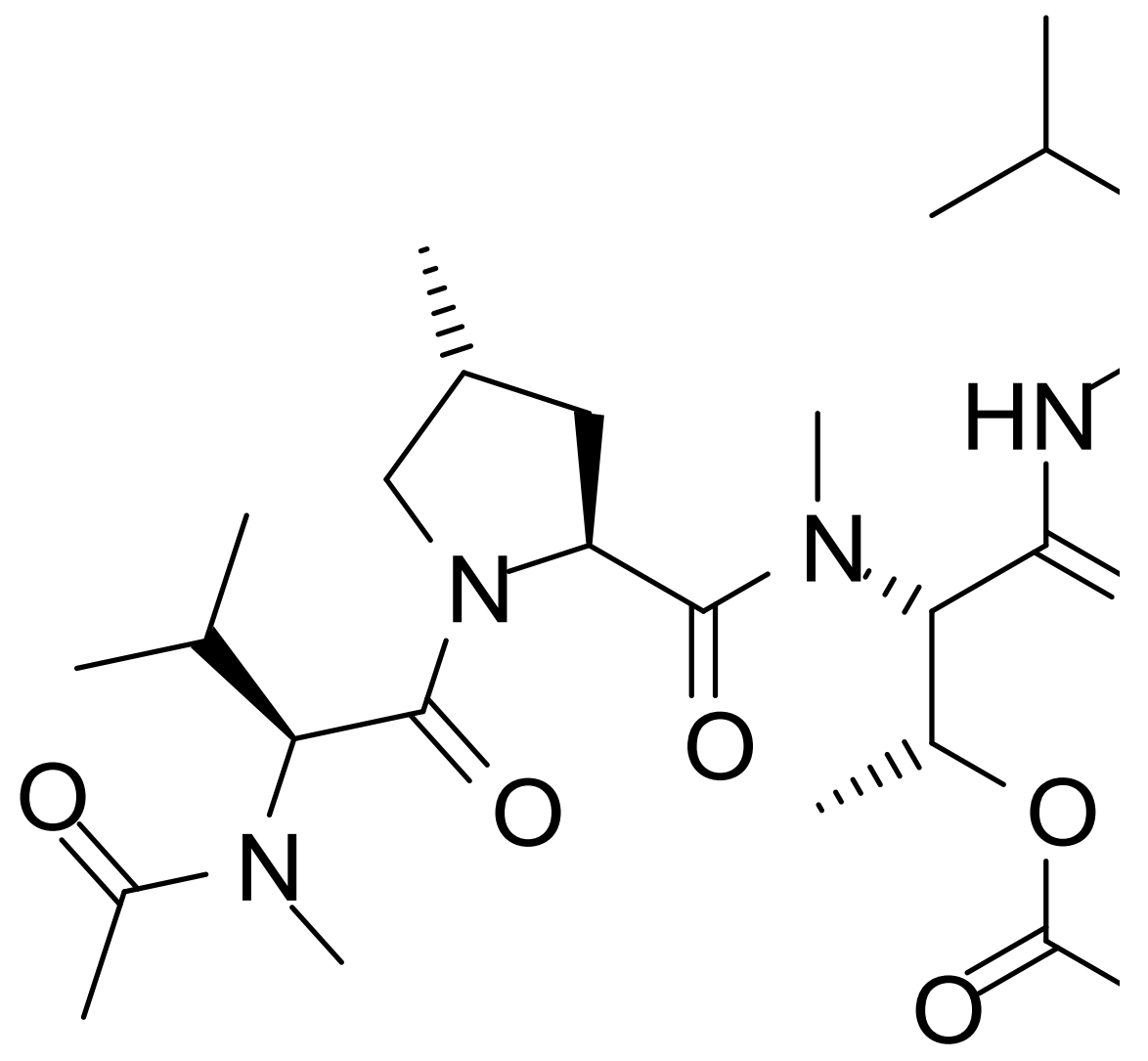

Griselimycin(1):

Cyclohexylgriselimycir

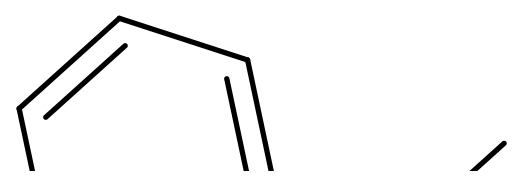

